\title{
Beitrag zur Lehre von der Hydroa aestivalis.
}

\author{
Von
}

Prof. Scholtz (Königsberg).

Im vergangenen Sommer hatten wir Gelegenheit in der Poliklinik einen Krankheitsfall von Hydroa aestivalis zu beobachten, welcher nicht nur wegen der erst zweimal beobachteten Miterkrankung der Conjunctiva und Cornea von besonderem Interesse war, sondern uns auch Gelegenheit zu histologischen und experimentellen Untersuchungen gab.

Die Krankengeschichte ist kurz folgende:

Fritz Meller, 21 Jahre alt, Besitzerssohn. Hereditär nicht belastet. Nach den Angaben des Patienten ist im Anschluß an die erste Impfung im ersten oder zweiten Lebensjahre ein Ausschlag über den ganzen Körper aufgetreten, welcher mehrere Monate bestanden hat. Über das Aussehen des Ausschlags ist nichts näheres zu eruieren. Unter der Behandlung mit Teersalbe ist der Ausschlag auf dem Rumpf allmählich besser geworden, auf dem Gesicht, den Händen und Fïßen ist er dagegen entweder überhaupt nicht abgeheilt oder es ist hier bald darauf von neuem Ausschlag aufgetreten. Nähere bestimmte Angaben können Pat. und dessen Eltern hierüber nicht mehr machen. Dagegen steht fest, daß schon vom dritten Lebensjahr ab im Frühling nur auf dem Gesicht, den Handrücken und FuBrücken ein Ausschlag auftrat, welcher den Rumpf vollständig freiließ, im Herbst allmählich auch auf den erkrankten Körperstellen abheilte und im Winter gaaz fortblieb, in jedem Frühjabr dagegen regelmäßig wieder von nenem erschien. Der Ausschlag wird als vorwiegend blasig bezeichnet und soll in der Kindheit so stark gewesen sein, daß Patient nicht gehen und die Hände nur wenig benutzen konnte. Im Gesicht waren besonders die Ohren, Nase und Wangen ergriffen, aber auch die A ugen waren beijedem Ausbruch der Krankheit stark gerötet und geschwollen, so daB Patient als Kind oft gar nicht sehen konnte. Später soll der Ausschlag von Jahr zu Jahr in geringerem Maße, sonst aber im wesentlichen in gleicher Form aufgetreten sein; nur die Blasenbildung war später nicht mehr so stark, sondern es traten mehr klenie 
derbe Blasen und Knötchen auf. Als Kind und als Knabe ging Patient im Sommer in der Regel barfuß, aber bereits im beginnenden Knabenalter hörte der Ausschlag auf den Fußrücken vollständig auf und auch jetzt kann Patient selbst im heißen Sommer barfuß gehen, ohne daß sich der Ausschlag auf den Füßßen zeigt. Gesicht, Handrücken und Augen werden dagegen noch jetzt regelmäßig jedes Frühjahr von dem geschilderten Ausschlag befallen.

Den Verlauf der Krankheit in den letzten Jahren schildert der Kranke etwa folgendermaßen: Gewöhnlich im April oder Anfang Mai, wenn ich viel auf dem Felde arbeite, tritt der erste Schub des Ausschlags teils in Form einzelner, wasserhaltiger, bis hellergroßer Blasen, teils in Form hochroter derber Knötchen im Gesicht und auf den Handrücken auf. Die Handflächen bleiben stets verschont, ebenso die Füße und der Rumpf. Gewöhnlich röten sich zu gleicher Zeit die Augen besonders stark entzündet sich das linke Auge, so daB ich schlecht sehen kann. Die Blasen platzen gewöhnlich sehon nach $2 t$ Stunden und die Stelle bedeckt sich dann mit einem Schorf, der in der Regel erst nach ca. 8 Tagen abfällt und eine gerötete Stelle hinterläßt. Aus den Knötchen bilden sich zum Teil nach 24-48 Stunden kleine Bläschen, zum Teil trocknen sie ein und wandeln sich gleich in einen testhaftenden Schorf um.“

Besondere Beschwerden und Jucken verursachen die frischen Blasen oder Knötehen nicht, und auch das Allgemeinbefinden ist nach Angabe des Kranken während des Ausschlags kaum gestört.

Status und Verlauf: Der etwas stupide Kranke hat kräftigen Knochenbau, er befindet sich in mäßigem Ernährungszustande, die Haut ist etwas welk und trocken.

Die Körperhaltung ist etwas gebückt. Herz, Lungen, Leber, Milz ohne besonderen Befund. Im Urin ca. 1.4\% Albumen, im Sediment vereinzelte granulierte Zylinder.

Die Haut des Gesichtes erscheint im ganzen narbig verändert und bunt gefleckt. Bei genauerer Untersuchung läßt sich folgender Befund erheben: Die Nase ist im ganzen atrophisch, verschmächtigt; die Haut über der Nase ist ziemlich gleichmäßig narbig verändert, mit dem Knorpel und Knochen fest. verwachsen und teils mehr rot, teils weiß gefärbt; an der Nasenwurzel wird die Haut im ganzen wieder normal und es finden sich hier nur noch isolierte, ca. linsengroße, ziemlich scharf umschriebene, leicht eingesunkene Narben. Ebenso sieht man auf der Haut der Wangen teils mehr diffuse narbig-atrophische Stellen, teils scharf umschriebene dellenartige Narben wie nach Pocken. Zwischen diesen Narben, aber auch auf den alten 
Narben selbst findet man dann stecknadel- bis kleinerbsengroße hochrote derbe Papeln, welche im Zentrum teils ein Bläschen, teils einen trockenen festhaftenden Schorf tragen, nach dessen Ablösung eine feuchte Delle oder bei den älteren Effloreszenzen eine vertiefte Narbe zurückbleibt. Die Effloreszenzen gleichen in der Form wie Entwicklung also teils den Effioreszenzen eines Erythema papulatum, teils denen einer Acne necrotisans. Daneben findet sich noch eine etwas größere mehr impetiginöse Borke auf einer Wange, welche von einer größeren Blase herrühren soll. Das Bild wird noch bunter durch das Bestehen unregelmäßig verteilter und unregelmäßig geformter brauner Pigmentationen von Stecknadelgröße bis fast Hellergröße, die vornehmlich die gesunden, zum Teil aber auch die narbig veränderten Hautstellen bedecken. Irgend ein direkter Zusammenhang zwischen den Narben und diesen "Sommersprossen" oder letzteren und den frisch auftretenden Efloreszenzen besteht offenbar nicht.

Die Ohren sind ebenfalls stark narbig verändert, der Helix und das Ohrläppchen sind ganz atrophisch, wie abgegriffen und narbig eingekerbt, die Haut im ganzen gerötet, narbig-glänzend und mit dem Knorpel fest verlötet.

Um es möglichst kurz zu charakterisieren, so gleichen die narbigen Veränderungen an der Nase und besonders an den Ohren in hohem Maße den Narben und Atrophien, wie wir sie nach Lupus erythematodes zu sehen gewohnt sind; die kleinen dellenförmigen Narben entsprechen ganz dem Aussehen von Pockennarben und die unregelmäßigen mehr diffusen atrophischen Stellen auf den Wangen in Verbindung mit den Pigmentationen erinnern an das Bild des Xeroderma pigmentosum. Die ganz frischen papulösen Effloreszenzen sind, wie schon erwähnt, urticariell-exsudativ und erinnern am meisten an die Effloreszenzen des Erythema papulatum, während sie nach 1-2 Tagen infolge von trockener Nekrosenbildung im Zentrum und Nachlaß der entzündlichen Erscheinungen mehr den Effloreszenzen der Acne necrotisans gleichen.

Im Prinzip ganz identische Veränderungen wie im Gesicht finden sich an der Haut der Handrücken und über der Streckseite der Handgelenke, nur fehlen hier die bräunlichen Pigment- 
flecken und die Effloreszenzen sind infolge ziemlich erheblicher venöser Stauung mehr bläulich-rot gefärbt. Erkrankt ist hauptsächlich die Partie über dem Metacarpus; hier ist die Haut vollständig narbig, depigmentiert und keloidartig verdickt. In gleich starker Weise ist die Haut über den Endphalangen ergriffen, während sich auf den übrigen Partien des Handrückens und über der Streckseite des Handgelenkes nur vereinzelte pockenähnliche Narben und frische Effloreszenzen finden. Auch auf den Händen sind die frischen Effloreszenzen in der großen Mehrzahl exsudativ-urtikariell und es finden sich nur zwei reichlich linsengroße wirkliche Blasen.

Die Vergleichung der frischen und älteren Effloreszenzen und vor allen Dingen die während der Beobachtungszeit des Patienten entstandenen Nachschübe ergaben, daß die Effloreszenzen sich in folgender Weise entwickeln: Ziemlich rasch etwa innerhalb von 12 Stunden treten derbe exsudative Knötchen auf, welche im ganzen den Effloreszenzen des Erythema papulatum entsprechen. Innerhalb der nächsten 12-24 Stunden bildet sich bei einem Teil dieser Knötchen im Zentrum ein kleines, kaum stecknadelkopfgroßes Bläschen, während größere, etwa hanfkorn- oder linsengroße Blasen nur selten auftreten. Bei anderen Knötchen ist überhaupt keine Blasenbildung nachweisbar. Gewöhnlich schon am dritten Tage ibres Bestandes bildet sich im Zentrum der Knötchen ein kleiner trockener Schorf, sei es nun, daß das zentrale Bläschen, ohne zu platzen, eintrocknet und es dadurch zur Schorfbildung kommt, sei es, daß der Schorf, ohne vorherige Bläschenbildung durch trockene Nekrose des Knötchenzentrums entsteht. In den folgenden 24-48 Stunden nimmt die Größe des Schorfes noch zu und erreicht bisweilen Linsengröße. Der Schorf haftet anfänglich sehr fest und ist nur unter leichter Blutung zu lösen. Nach drei bis vier Tagen fällt er spontan $a b$ und hinterläBt eine gedellte rötliche Narbe. Wie dies schon bei den meisten früheren Fällen beobachtet worden ist, traten auch bei unserem Kranken Nachschübe hauptsächlich auf den am meisten veränderten Hautstellen auf, während die noch intakte Haut des Gesichtes und der Handrücken nur selten von einzelnen meist kleineren Knötchen befallen wurde. 
Von besonderem Interesse war bei unserem Kranken die Beteiligung der Conjunctiva und Cornea an der Erkrankung. Jedesmal zu gleicher Zeit mit dem Hautausschlag trat eine ziemlich starke Conjunctivitis mit intensiver ciliarer Injektion und Bildung kleiner Phlyktänen auf und auch auf der Cornea kam es zu oberflächlichen Epithelverlusten. Gleichzeitig bestand leichte Rötung und Schwellung der Augenlider und erhebliche Lichtscheu. Jedesmal mit Rückgang des Hautausschlages schwanden 'auch die Erscheinungen von Seiten des Auges und es blieben als Residuen der Augenerkrankung nur hie und da leichte rauchige Trübungen der Cornea zurück, welche sich erst laggsam wieder aufhellten. Nur zwei gut stecknadelkopfgroße, weißliche Trübungen der Cornea sind links wohl schon von der Kindheit her stabil geblieben. Identische Beobachtungen sind bekanntlich von Möller und Ledermann gemacht worden, und auch in dem Falle von Halberstädter dürfte eine gleiche Erkrankung der Conjunctiva und Cornea vorgelegen haben. Die etwas gebückte Haltung, welche unser Patient jetzt dauernd sich angewöbnt hat, ist zweifellos durch die mit der Conjunctivitis verbundenen Lichtscheu bedingt worden. Ähnliches ist ja beim Xeroderma pigmentosum beobachtet worden.

Um den Hautausschlag wie die Augenerkrankung zur Abheilung zu bringen, genügte es bei unserem Patienten denselben etwa drei Tage in einem wenig erleuchteten Zimmer zu halten. Sobald derselbe sich aber nur wenige Tage bei der Feldarbeit etc. der Sonne ausgesetzt hatte, trat sofort ein Rezidiv auf. Wir haben diese Beobachtung bei unserem Pat. zweimal machen können. Leider ist der Albumengehalt des Urins nur bei dem letzten klinischen Aufenthalt des Patienten genau verfolgt worden, wobei sich herausstellte, da $B \mathrm{mit}$ dem Erlöschen des Ausschlages auch das Albumen im Urin verschwand. Es würde dies ein Analogon zu den Beobachtungen von Möller, Linser u. a. sein, welche feststellten, daß eine bei dem Patienten zur Zeit des Ausschlages bestehende Hämatoporphyrinurie mit Erlöschen des Ausschlages jedesmal verschwand.

Es war uns bei unserem Patienten auch möglich, eine 
histologische Untersuchung frischer 12-24 Stunden alter Effloreszenzen vorzunehmen. Wir haben dabei die Beobachtung von Malinowsky, wonach das Primäre Fpithelnekrose, das Sekundäre Entzündung sein soll, in keiner Weise bestätigen können, sondern sind zu dem gleichen Resultat wie frühere Autoren, speziell Bow en und Mibelli gekommen.

Wir fanden in den jüngsten Effloreszenzen stets außerordentlich lebhafte Entzündungsvorgänge im Corium und in der Epidermis. In der Cutis und im Papillarkörper sahen wir hochgradige Gefäßerweiterung, sogar kleine Blutungen, starkes Ödem, massenhafte Emigration von Leukocyten und Rundzelleninfiltration. Häufig war die Exsudation so stark, daß das Epithel in toto durch das flüssige Exsudat vom Papillarkörper abgehoben und zwischen Epithel und Papillarkörper ein mit Serum gefüllter Spalt entstanden war. Das Epithel selbst war an den erkrankten Stellen stets stark ödematös, von Rundzellen und Leukocyten durchsetzt, die Zellen gequollen, auseinandergezogen, schlecht färbbar, ja häufig das ganze Epithel schon in toto nekrotisch. A ber überall war doch deutlich zu erkennen, daß die EntzündungdasPrimäre, die Nekrose das Sekundäre ist, wenn letztere infolge des starken Ödems und der vollständigen Abhebung des Epithels auch sehr rasch, ja auffallend rasch eintritt.

Ferner haben wir bei unserem Patienten einige Versuche vorgenommen, um festzustellen, was das wirksame A gens bei Entstehung der Hydroa vacciniformis ist. Daß die Sonnenbestrablung eine wesentliche Ursache, mindestens das auslösende Moment der Erkrankung darstellt, das ist ja von jeher angenommen worden und steht nach den klinischen Beobachtungen, nach denen in typischen Fällen stets nur die unbedeckten Körperpartien zu Zeiten starker Besonnung ergriffen werden, außer Zweifel. Welche Iichtstrahlen dabei aber wirksam sind, ob die kurzwelligen chemisch wirksamen oder die langwelligen wesentlich Wärme erzeugenden Strahlen. das ist noch immer zweifelhaft. Bekanntlich ist erst vor wenigen Monaten Ehrmann auf Grund von scheinbar recht beweisenden Experimenten nachdrücklichst dafür eingetreten, 
daß es ausschließlich die kurzwelligen Strahlen sind, welche die Hydroa hervorrufen.

Unsere Versuche wurden an unserem Patienten am Unterarm teils an ganz gesunden Hautpartien, teils an Hautstellen in der Nähe des Handgelenkes vorgenommen, wo schon vereinzelt Narben von Hydroa-Effloreszenzen vorhanden waren. Letztere Hautstellen wählten wir deshalb zu unseren Versuchen, da von Möller und Linser angegeben worden ist, daB nur die von der Hydroa befallenen Hautpartien eine besondere Empfindlichkeit gegen Lichtstrahlen zeigen. Wir haben die Bestrahlungen teils mit Eisenlicht (Eisenelektrodenlampe von Reiniger, Gebbert und Schall), teils mit Kohlenlicht (Tripletlampe der Sanitasgesellschaft mit Konzentrationslinse) vorgenommen und teils ohne Filter, teils mit Anwendung von roten und blauen Glasfiltern und Flüssigkeitsfiltern von Karminrespektive Kupferoxydammoniak-Lösungen bestrahlt. W i $\mathrm{r}$ h a b e n ebenso wie Halberstädter u. a. bei keinem dieser Versuche irgend eine abnorm hohe Empfindlichkeit gegen chemisch wirksame Strahlen nachweisen können; wie Kontrollversuche ergaben, hielt sich die Empfindlichkeit der Haut gegen ultraviolette, violette und blaue Lichtstrahlen bei unserem Patienten stets in durchaus normalen Grenzen und die Reaktion trat in durchaus gleicher Weise wie bei normalen Personen auf, das heißt nach Anwendung der Eisenlampe oder der Finsenlampe erschien nach 12-24 Stunden ein Erythem eventuell Blasenbildung, welche sich dann in üblicher Weise veränderten. Bedingung war bei diesen Versuchen, daß auch die leiseste Erwärmung verhindert wurde. Der Patient durfte auch nicht das geringste Brennen während des Versuches empfinden. Rief die Bestrahlung auch nur leichtes Brennen hervor, dann traten allerdings ganz identische Veränderungen auf, wie sie von Ehrmann beschrieben worden sind, das heißt, es zeigte sich schon nach 10 Minuten eine entsprechend große, im Zentrum oft leicht gedellte Quaddel mit diffus rotem Hofe, die sich in den nächsten Tagen in einen Schorf umwandelte und dann unter Narbenbildung abheilte. Daß das Gleiche auch bei normalen Personen eintritt, wissen wir zur Genüge von den Finsenbestrahlungen 
her. Wird bei diesen jede für den Patienten fühlbare Erwärmung vermieden, so tritt die Reaktion stets erst nach ca. 12 Stunden in Form von Erythem und Blasenbildung auf, kommt es dagegen z. B. durch ungenügende Kühlung auch nur zu leichtem Brennen, so zeigt sich wie in den $\mathrm{E} \mathrm{hr} \mathrm{m} \mathrm{an} \mathrm{n}$ schen Versuchen schon nach 10-15 Min. Quaddelbildung mit nachfolgender Nekrose. Auf Grund dieser Tatsache und unserer Beobachtungen glauben wir, daß d e $\mathrm{Ersch}$ e in ung e n bei den Ehrmannschen Experimenten ebenfalls a uf Wärmeentwicklung zurückzuführen sind, und bei der Hydroa vacciniformis eine Überempfindlichkeit gegen die kurzwelligen, rein chemisch wirkenden Lichtstrahlen nicht besteht.

Eher sprechen unsere Versuche dafür, daß Patienten, welche an Hydroa leiden, gegen die langwelligen, leuchtenden Lichtstrahlen, welche bei ihrer Absorption wesentlich Wärme erzeugen, besonders empfindlich sind. Wenigstens sahen wir bei gleicher Beleuchtung an uns selbst. wobei nur ganz leich te Wärmeempfindung auftrat, nur weit geringere Reaktionserscheinungen auftreten. Dab auch die Wärmeentwicklung infolge der Belichtung resp. Besonnung bei Entstehung der Hydroa eine Rolle spielen dürfte; dafür würde auch die Beobachtung sprechen, daß die Hydroa so gut wie ausschließlich zur warmen Jahreszeit auftritt, während im Winter bei Wanderungen über Schneefelder der Ausschlag nicht oder nur ganz ausnahmsweise erscheint, obwohl die Lichtwirkung bekanntlich dabei eine besonders intensive ist. ${ }^{1}$ ) Wir sind jedoch weit davon entfernt, auf Grund dieser Beobachtung etwa zu behaupten, daß die langwelligen leuchtenden Lichtstrahlen - vielfach als Wärmestrahlen bezeichnet - die Ursache der Hydroa wären, im Gegenteil wir glauben, daß der ganze Mechanismus bei der Entstehung

1) Wir müssen uns bei der Einteilung des Lichtes in chemisch wirksame Strahlen und Wärmestrahlen stets bewußt sein, daß es sich dabei nicht um prinzipiell verschiedene Strahlen handelt, sondern daß. wir nur sagen können, die einen Strahlen rufen bei ihrer Absorption wesentlich Wärme hervor, die anderen haben mehr chemische Wirkung. Auch darf nicht nur die Wellenlänge der Strahlen in Betracht gezogen werden, sondern auch die Art und Weise, wie die Strahlen von dem betreffenden Medium absorbiert werden, muß berücksichtigt werden. 
der Hydroa überhaupt nicht ganz so einfach ist, wie man gewöhnlich annimmt, und daß sich das Auftreten des eigenartigen Exanthems mit einer einfachen Überempfindlichk eit der betreffenden Patienten gegen Lichtstrablen, seien es nun kurz-oder langwellige, nicht erklären läßt. Läge eine einfache Überempfindlichkeit gegen Lichtstrahlen vor, so könnte wohl ein einfaches Erythem auftreten, wie wir es vom Gletscherbrand oder von der Behandlung mit Eisenlicht etc. her kennen, aber es könnten nicht derartige eigenartige isolierte Effloreszenzen erscheinen. Wir dürfen mit Möller in der Besonnung wohl nur das auslösende Moment für die Dermatose sehen und müssen die eigentliche Krankheit oder die eigentliche Ursache für die Erkrankung der Haut in einem eigenartigen Zustand des Gesamtorganismus vielleicht einer Art Intoxikation suchen. Um das Wesen der Hydroa recht zu verstehen, müssen wir dieselbe in Parallele zur Pellagra, zu der Urticaria der Schweine an pigmentlosen Körperstellen nach Buchweizenfütterung etc. stellen und dürfen sie nicht mit dem Erytheme solare oder caloricum vergleichen, wie dies vielfach ( $\mathrm{Ehr}$ mann, J os e ph Lehrbuch etc.) geschieht. Fingerzeige dafür, daß auch bei der Hydroa, öfter als früher angenommen wurde, Erscheinungen von seiten des gesamten Organismus vorliegen, haben wir durch die in den letzten Jahren publizierten Beobachtungen über das gleichzeitige Auftreten und Verschwinden von Hämatoporphyrin im Urin mit dem Ausbruch und Erröschen des Hautausschlages schon bekommen. Auch in unserem Falle dürfte das Verschwinden des Albumen im Urin mit Erlöschen des Exanthems ähnlich gedeutet werden können. Natürlich sind wir uns bewubt, daß möglicherweise die Urinveränderungen auch nur sekundär durch die Hauterkrankung bedingt sein kann.

Immerhin wird in künftigen Fällen auf derartige Urinbefunde etc. genau zu achten und ihre Beziehungen zu der Hautaffektion nach Möglichkeit zu eruieren sein. Es kommt für das Verständnis der Hydroa aestivalis weniger darauf an, nachzuweisen, welche Strahlen des Spektrums es sind, unter deren Einwirkung das Exanthem auftritt, als festzustellen, welche abnormen Zustände des Gesamtorganismus oder der Haut bei den Kranken mit Hydroa das Auftreten des eigenartigen 
Exanthems unter der Einwirkung der Lichtstrahlen bedingen. Um eine einfache Überempfindlichkeit der Haut gegen Lichtstrahlen handelt es sich jedenfalls nicht.

Sehr schwierig ist bekanntlich auch die Frage der Abgrenzung der typischen Hydroa gegen Exantheme, welche der Hydroa hinsichtlich der Form gleichen und ebenfalls vornehmlich im Sommer auftreten.

Derartige Exantheme sind bekanntlich unter dem Namen Sommerprurigo, Dermatitis papulo-vesiculosa solaris (Möller), schon wiederholt beschrieben worden. Auch wir haben kürzlich einen Fall beobachtet, welcher in diese Krankheitsgruppe gehört. Es handelte sich um eine 39jährige Frau, welche seit $8 \mathrm{Jahren}$ an Ausschlägen des Gesichtes, Halses, der Handrücken und der Vorderarme leidet. Hals und Vorderarme werden von der Patientin frei getragen. In den ersten Jahren trat dieser Ausschlag ganz wie die echte Hydroa erst im Frühjahr auf, hielt dann den Sommer über an und versehwand Anfang des Winters. In den letzten Jahren verschwindet der Ausschlag aber auch im Winter nicht mehr vollständig sondern bessert sich nur etwas. Sowohl nach der Beschreibung der Kranken als nach unsern eigenen Beobachtungen gleicht dieser Ausschlag auch in seiner Form in vieler Hinsicht der typischen Hydroa. Vor allen Dingen hat er vielfach auf der Stirn, den Wangen und dem Halse zu pockenartigen Narben wie die echte Hydroa geführt, und auch die Knötchen, mit denen das Exanthem gewöhnlich beginnt, ähneln in hohem Grade den Knötchen bei typischer Hydroa. Es unterscheidet sich aber die Erkrankung von echter Hydroa nicht nur daduroh wesentlich, daß das Exanthem erst mit 31 Jahren aufzutreten begann und jetzt auch im Winter nicht mehr vollständig abheilt, sondern vor allem auch dadurch, daß jetzt stets ausgesprochene ekzematöse Entzündung der ergriffenen Hautpartien hinzutreten und bisweilen auch ziemlich starkes Nässen eintritt.

Daßj Lichtwirkung bei der Erkrankung eine erhebliche Rolle spielt, lieb sich dadurch erweisen, daß sich der Ausschlag nach dem Tragen dichter bräunlicher Schleier oder dauernden Einfettungen mit Licht abhaltenden Salben wesentlich besserte, Eine Üherempfindlichkeit gegen ultraviolette und violette Lichtstrahlen war auch hier nicht; nachweisbar, während bei intensiver Belichtung so daß leichte Wärmeewirkung auftrat, sich wiederum eine auffallend intensive Reaktion einstellte. Irgendwelche Erkrankungen innerer Organe, speziell der Nieren waren bei der Pat. nicht nachweisbar.

Wir sind weit entfernt diesen Fall mit echter Hydroa identifizieren zu wollen, aber wir erwähnen ihn, da er wieder zeigt, daß es auch Exantheme von mehr ekzematösem Charakter gibt, die nicht nur in der Lokalisation und im Aussehen sondern auch in ihrem Auftreten nnd in jhrem Verlauf Ähnlichkeit mit echter Hydroa haben. 